\title{
Sphingosine 1-Phosphate
}

National Cancer Institute

\section{Source}

National Cancer Institute. Sphingosine 1-Phosphate. NCI Thesaurus. Code C126907.

A bioactive sphingolipid comprised of a sphing osine with a phosphate group attached to the carbon at position 1. Sphing osine 1-phosphate (S1P) binds to G protein-coupled S1P receptors (S1PRs) and initiates signaling pathways involved in the regulation of angiogenesis, the proliferation of skin cells, vascular stability and permeability, and T- and B-cell trafficking. 